\title{
Transfusion Medicine Practice During COVID-19 Pandemic: Potential Pitfalls and Solutions for Provision of Blood Products
}

Sarah Tehseen

Saskatchewan Health Authority, Canada CA.
$\mathrm{T}$ ransfusion of blood components is an essential part of clinical medicine and can be significantly impacted by pandemics. Therefore, hospital blood transfusion services must plan for the potential shortage of blood supply to continue the provision of this critical service in the face of emergencies. ${ }^{1}$ This article focusses on recommendations to ensure a safe and reliable supply of blood components.

Impact of a pandemic on the blood supply chain can be observed in multiple areas including the potential loss of donors, lack of reagent red cells or other materials needed for pre-transfusion testing, staff shortages due to illness and increased utilization by the hospital due to an increase in the number of sick patients. $^{2}$

\section{Channels of Communication:}

It is paramount that the blood transfusion services of each hospital review its inventory and communicate with the blood suppliers frequently to review the status of blood availability. Frequent communication with patient care providers (anesthesia, oncology, medicine, etc.) to estimate the daily need for blood products is also critical. It is also crucial for each hospital to minimize blood utilization where possible and develop contingency plans for severe shortages. ${ }^{3}$

\section{Reducing Hospital Utilization:}

A large volume of high-quality data both in adults and pediatrics strongly favors restrictive transfusion thresholds in hospitalized patients in the absence of active hemorrhage, hemolysis or trauma., ${ }^{4,6}$ Enforcement of these restrictive thresholds (Table1 a $\& 1$ b) will ensure a reduction in blood utilization and maintain blood inventory. ${ }^{7}$ This can be done by review of transfusion orders by the blood bank (Table-2) as well as the widespread education of healthcare providers with a focus on addressing the cause of underlying anemia (Table-1a). Also, postponing elective procedures and surgeries will help reduce blood utilization during an active pandemic. $^{3}$
Other considerations include blood conservation strategies like normovolemic hemodilution and the use of cell savers during surgery. Optimization of surgical control of bleeding and the use of antifibrinolytic agents such as tranexamic acid also plays an important role in reducing the need for blood transfusion. ${ }^{8,9}$

Massive hemorrhage ( $>4.5$ liters of blood loss in 30 minutes OR $150 \mathrm{ml} / \mathrm{min}$ of blood loss OR > anticipated need of 3 or more red cell units in 3 hours) requires fast delivery of multiple blood components and can deplete inventory quickly. ${ }^{18}$ It is recommended to initiate a conversation with the healthcare providers early in the situation of a massive hemorrhage to anticipate the need for blood components. Switch to type-specific blood as soon as possible to protect the $\mathrm{O}$ negative inventory and consider the use of antifibrinolytics and coagulation factor concentrates such as fibrinogen and prothrombin concentrates if available. ${ }^{10}$

\section{Staff and Testing Supply Shortages:}

Shortage of staff personnel and testing supplies in the light of an ongoing pandemic must be considered. Hospitals should have contingency plans for staff coverage should many technologists fall sick. Remote working should be considered for medical directors whenever possible. At this time there are no specific recommendations for protective equipment for blood bank laboratory technologists beyond handwashing, social distancing, and universal precautions however in the light of the dynamic situation of the current pandemic, these may be reconsidered. ${ }^{11}$

Hospitals should also have contingency plans in light of a shortage of testing reagents. For example, if testing reagents for pre-transfusion testing fall short, consider limiting to basic testing such as $\mathrm{ABO}, \mathrm{RH}$ and cross-matching for transfusions.

\section{Specific Issues in Developing Countries:}

Pakistan lacks a centralized system or coordinated blood transfusion service leading to wide heterogeneity in terms of availability of blood 
components, the sufficiency of blood supply, presence and educational level of medical directors and technologists as well as infrastructure and other resources. ${ }^{12}$ While the blood supply for patients with hemoglobinopathies come from volunteer donors, most rural and small city clinics and hospital rely on whole blood donation from patients' relatives and friends in cases of surgeries or other procedures which raises significant questions about the quality and safety of blood components. It is, therefore, critical that hospital systems providing care at various levels, collaborate to achieve the following:

1. Sharing blood inventory details across different hospitals and providing components to centers in demand.

2. Formulating and enforcing restrictive transfusion strategies and adequate hemorrhage control

3. Contingency planning for shortages in staff and testing reagents with a delineated centralized hospital where specimens may be referred for advanced transfusion testing if needed

\section{Blood Collection Perspective:}

Given the anticipated increase in need of blood for patients, there should be national and provincial level initiatives for blood donations, especially to maintain the blood supply for patients with hemoglobinopathies. This is complicated in the light of social distancing recommendations which are crucial to prevent the spread of COVID-19 infections. Donor centers should consider the following measures for donor and staff protection

1. Screen donors with questions regarding cough and fever before accepting them for donation to prevent droplet/airborne spread of the virus in collection centers.

2. Avoiding crowding by maximizing donor appointments, staggering appointments, and offering donors the opportunity to wait in their cars or other convenient locations.

3. Discouraging children \& others from accompanying donors to blood drives.

4. Using signs outside blood drives asking individuals at elevated risk for infection (due to travel, illness or contact with sick individuals) not to enter/donate.

5. Donors and staff wear surgical masks. ${ }^{13}$

There is no current evidence that SARS-COV2 is transmissible by blood however the measures mentioned above are crucial to prevent the spread of infection among donors and staff personnel performing blood collection.
Do not transfuse for anemia if other therapies or observation is effective

Limit transfusion to one unit at a time in a stable nonbleeding patient

Avoid plasma transfusion for a mildly elevated INR $(<1.8)$ in a nonbleeding patient or before a procedure for INR correction

Do not order pre-transfusion testing for all preoperative patients unless significant bleeding is anticipated

Limit transfusion of $\mathrm{O}$ negative units to women of childbearing potential for emergencies and to patients who have $\mathrm{O}$ negative blood type

Table-1a: Recommendations for Red cell transfusion based on choosing wisely Canada ${ }^{14,15,16}$

\begin{tabular}{|l|l|}
\hline $\begin{array}{l}\text { Hemoglobin } \\
>9 \mathrm{~g} / \mathrm{dl}\end{array}$ & $\begin{array}{l}\text { Mostly inappropriate unless special } \\
\text { circumstances. }\end{array}$ \\
\hline $\begin{array}{l}\text { Hemoglobin } \\
8-9 \mathrm{~g} / \mathrm{dl}\end{array}$ & $\begin{array}{l}\text { Transfusion likely inappropriate unless } \\
\text { symptomatic anemia (tachycardia, } \\
\text { CNS symptoms, hypotension). }\end{array}$ \\
\hline $\begin{array}{l}\text { Hemoglobin } \\
7-8 \mathrm{~g} / \mathrm{dl}\end{array}$ & $\begin{array}{l}\text { One unit appropriate for transfusion in } \\
\text { cardiac patients. }\end{array}$ \\
\hline $\begin{array}{l}\text { Hemoglobin } \\
<7 \mathrm{~g} / \mathrm{dl}\end{array}$ & $\begin{array}{l}\text { Usually appropriate to transfuse 1 } \\
\text { RBC or whole blood unit followed by } \\
\text { checking hemoglobin UNLESS: } \\
1 . \text { The patient has chronic iron } \\
\text { deficiency anemia (IDA) where IV } \\
\text { iron is a better alternative. }\end{array}$ \\
& 2. Young/pediatric patient. \\
\hline $\begin{array}{l}\text { Transfusion appropriate unless chronic } \\
<6 \mathrm{~g} / \mathrm{dl}\end{array}$ & \begin{tabular}{l} 
IDA. \\
\hline
\end{tabular}
\end{tabular}

Table-1b: Restrictive transfusion thresholds for nonbleeding stable patients ${ }^{17}$

$>1$ red cell/whole blood unit for orders outside of Operating room (OR)

$>4$ red cell units requested / case for surgeries

$>1$ platelet unit requested outside the OR

Initiation of discussion between TM physician/Blood bank and patient care provider in cases of massive hemorrhage to anticipate need for blood components

Table-2: Criteria for prospective order review to conserve inventory by the transfusion medicine physician or lab technologists ${ }^{13}$

\section{CONCLUSION}

The COVID-19 pandemic can have a significant impact on the ability of hospitals and blood systems to provide life-saving transfusions. In this light, preparation by enforcing restrictive transfusion thresholds, postponing elective procedures, physician education and contingency planning for staff and testing supply shortages can go a long way towards mitigating the adverse effects of blood shortages. The most crucial step in these measures is frequent communication between direct patient care providers and blood banks to review inventory and 
anticipate the need for blood products. This communication also needs to happen between hospitals in cities and provinces to address shortages in blood components.

\section{REFERENCES}

1. World Health Organization. Maintaining a safe and adequate blood supply during pandemic influenza. Available at:

https://www.who.int/bloodsafety/publications/

WHO_Guidelines_on_Pandemic Influenza and Blood_Supply.pdf

2. Zimrin, Ann B., and John R. Hess. "Planning for pandemic influenza: effect of a pandemic on the supply and demand for blood products in the United States." Transfusion 47.6 2007):1071-79

3. Pagano, Monica B., et al. "Prepare to adapt: Blood supply and transfusion support during the first 2 weeks of the 2019 Novel Coronavirus (COVID-19) pandemic affecting Washington State." Transfusion (2020).

4. Hébert, Paul C., et al. "A multicenter, randomized, controlled clinical trial of transfusion requirements in critical care." New England Journal of Medicine 340.6 (1999):409-17

5. Valentine, S. L., et al. "Pediatric Critical Care Transfusion and Anemia Expertise Initiative (TAXI)." Pediatric Critical Care Blood Research Network (Blood Net), and the Pediatric Acute Lung Injury and Sepsis Investigators (PALISI) Network PediatrCrit Care Med 19 (2018): 884.

6. Holst, Lars B., et al. "Restrictive versus liberal transfusion strategy for red blood cell transfusion: a systematic review of randomized trials with meta-analysis and trial sequential analysis." BMJ 350 (2015): h1354.

7. Carson, Jeffrey L., et al. "Red blood cell transfusion: a clinical practice guideline from the AABB." Annals of internal medicine 157.1 (2012): 49-58.

8. Henry, David A., et al. "Anti-fibrinolytic use for minimizing perioperative allogeneic blood transfusion." Cochrane database of systematic reviews 3 (2011).

9. Huët, Charlotte, et al. "A meta-analysis of the effectiveness of cell salvage to minimize perioperative allogeneic blood transfusion in cardiac and orthopedic surgery." Anesthesia \& Analgesia 89.4 (1999): 861.

10. Dzik, Walter H., et al. "Clinical review: Canadian national advisory committee on blood and blood products-massive transfusion consensus conference 2011: report of the panel." Critical Care 15.6 (2011): 242.

11. AABB townhall series: Prepare to adapt. The Washington state experience during COVID-19 pandemic. Pagano M. March 31 ${ }^{\text {st }}, 2020$.

12. Haddad, Antoine, et al. "How to manage transfusion systems in developing countries: The Experience of Eastern and Southern Mediterranean countries." Transfusion Medicine (2020).

13. AABB townhall series: Collection considerations during COVID-19 pandemic. Vassallo R, April 2 $2^{\text {nd }}, 2020$

14. Retter, Andrew, et al. "Guidelines on the management of anaemia and red cell transfusion in adult critically ill patients." British journal of haematology 160.4 (2013): 445-464.

15. Carson, Jeffrey L., et al. "Transfusion thresholds and other strategies for guiding allogeneic red blood cell transfusion." Cochrane database of systematic reviews 10 (2016).

16. Hillis, Christopher M., et al. "The Canadian Choosing Wisely campaign: the Canadian Hematology Society's top five tests and treatments." Annals of hematology 94.4 (2015): 541-545.

17. Screening algorithm for red cell transfusions. Saskatchewan Health Authority Saskatoon Canada.

18. Savage, Stephanie A., et al. "The new metric to define large-volume hemorrhage: results of a prospective study of the critical administration threshold." Journal of Trauma and Acute Care Surgery 78.2 (2015): 224-230.

\section{The Authors:}

Dr. Sarah Tehseen

Saskatchewan Health Authority, Canada CA.

Email: sarah.tehseen@gmail.com 